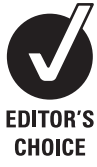

${ }^{1}$ Bobby R Alford Department of Otolaryngology-Head and Neck Surgery, Baylor College of Medicine, Houston, Texas, USA ${ }^{2}$ The Hearing Center at Texas Children's Hospital, Houston, Texas, USA; ${ }^{3}$ Department of Neuroscience, Baylor College of Medicine, Houston, Texas, USA;

${ }^{4}$ Department of Bioengineering, Rice University, Houston, Texas, USA

Correspondence to: Dr J S Oghalai, Bobby R Alford Department of OtolaryngologyHead and Neck Surgery, Baylor College of Medicine, One Baylor Plaza, NA-102, Houston, TX 77030, USA; jso@bcm.edu

Accepted 8 July 2008

\title{
Hearing loss in children with very low birth weight: current review of epidemiology and pathophysiology
}

\author{
R Cristobal, ${ }^{1}$ J S Oghalai $1,2,3,4$
}

\section{ABSTRACT}

An association between birth weight $<1500 \mathrm{~g}$ (very low birth weight (VLBW)) and hearing loss has been long recognised. As universal hearing screening programmes have become widely implemented and the survival rate of VLBW babies in modern intensive care units has increased, we have gained a substantially better understanding of the nature of this problem. However, many gaps in our knowledge base exist. This review describes recent data on hearing loss in the VLBW population and explains the current level of understanding about the physiological basis underlying the auditory deficits in these patients. Although VLBW alone may not have a severe impact on hearing, it is commonly associated with multiple other risk factors that can alter hearing in a synergistic fashion. Therefore, the risk of hearing loss is substantially higher than in the general newborn population. Also, it is important to perform a more comprehensive audiometric evaluation than standard otoacoustic emission screening for infants who are in the neonatal intensive care unit in order not to miss hearing loss due to retrocochlear pathology. Furthermore, children with VLBW are also at increased risk of experiencing progressive or delayed-onset hearing loss, and thus should continue to have serial hearing evaluations after discharge from the neonatal intensive care unit.

The survival of very-low-birthweight (VLBW) infants has increased substantially over the past two decades because of multiple improvements in obstetrical and neonatal care. ${ }^{1}$ Even though a rational approach to reducing the risk of developing hearing loss in this population should be based on a sound scientific understanding of the pathophysiology, the association between VLBW and hearing loss remains poorly understood. Although our knowledge base about the causes of hearing loss has improved to some degree, a thorough understanding of the mechanisms underlying the development of hearing loss in VLBW infants is unfortunately lacking. A major impediment to studying these mechanisms of injury is that biopsies of inner ear tissue cannot be carried out on patients beginning to develop hearing loss because this would produce immediate total hearing loss. Also, current audiometric and radiological studies do not provide enough detail on subtle changes in the auditory system. Therefore, the most common research strategy for investigating normal inner ear physiology is the use of animal models; however, few animal models of hearing loss in VLBW infants or with other risk factors for hearing loss exist. This review summarises the epidemiology of hearing loss in VLBW infants and condenses the current level of knowledge on the causative factors and pathophysiology of the auditory system.

\section{NORMAL HEARING PHYSIOLOGY}

The process of normal human hearing requires proper function of the external ear, middle ear, inner ear (cochlea) and ascending brainstem pathways (fig 1A). The process of hearing is initiated as sound pressure waves travel through the external auditory canal and vibrate the tympanic membrane. The ossicular chain in the middle ear space then transmits the acoustic energy to the cochlea. There are three fluid-filled chambers within the cochlea (fig 1B). The scala tympani and scala vestibuli contain perilymph, a fluid similar to serum in which the predominant cation is sodium. These perilymphatic compartments are in electrical continuity with the rest of the body and so they are at $0 \mathrm{mV}$. The scala media contains endolymph, which is similar to intracellular fluid in that the predominant cation is potassium, and the calcium concentration is also lower than that typically found in extracellular fluid. Importantly, the endolymphatic compartment is electrically isolated from the other compartments in that there is an endocochlear potential of about $+90 \mathrm{mV}$ within the scala media. The electrical and chemical gradients between the endolymph and perilymph function to power the cochlea and are maintained by the stria vascularis.

The organ of Corti runs longitudinally along the length of the cochlea and contains one row of inner hair cells and three rows of outer hair cells which reside over the elastic basilar membrane. Longitudinal gradations in the mass and stiffness of the basilar membrane create regional tonotopic differences in vibratory properties and sound frequency sensitivity. Thus, the basal end of the cochlea responds to high-frequency sounds and the apical end to low-frequency sounds. The apical surfaces of the hair cells have stereocilia, which function to transduce mechanical acoustic energy into electrical energy. The inner hair cell stereocilia are in close approximation to the overlying tectorial membrane, and the outer hair cell stereocilia are attached to it. When a sound pressure wave is applied by the stapes to the oval window at the base of the cochlea, a travelling wave is generated that vibrates the basilar membrane maximally at the region tuned to the frequency of the sound stimulus. The vertical movements of the basilar and tectorial membranes generate shearing forces that deflect the hair cell stereociliary bundles.

Bending of the stereocilia opens mechanosensitive channels near their tips and allows the influx 
of cations from the endolymph into the hair cell. In inner hair cells, the resultant depolarisation triggers synaptic neurotransmission to afferent auditory neurons. In contrast, outer hair cells generate unique forces that modify the physical properties of the organ of Corti and lead to frequency-selective amplification of the inner hair cell response. ${ }^{2}$ Sound information travels through the auditory nerve to the cochlear nucleus, and follows an organised path along multiple brainstem nuclei, ultimately conveying a signal to the auditory cortex, which lies within the temporal lobe adjacent to the Sylvian fissure.

\section{CLINICAL ASSESSMENT OF HEARING IN NEWBORNS}

Hearing screening of healthy term newborns is most commonly performed by measuring otoacoustic emissions (OAEs). OAE testing is based on the concept that outer hair cells within the cochlea can generate force in response to incoming sounds. By stimulating outer hair cells with a click or with two simultaneous tones of slightly different frequencies, the forces generated by the outer hair cells can be detected with a microphone in the ear canal as an emitted sound, the OAE. As OAE testing assesses the ability of the outer hair cells to receive an incoming sound wave and produce force in response to it, only cochlear function is tested (fig 1A, step 2). Thus, problems with neurotransmission from inner hair cells to the auditory nerve, the auditory nerve itself, or with the ascending auditory brainstem pathways are not detected (fig 1A, step 3). However, OAE testing depends on sounds passing through the ear canal and middle ear twice. The incoming sound goes through in a forward direction (fig 1A, step 1), and the emitted sound goes through in a reverse direction. Thus, any ear canal or middle ear pathology will have double the impact on the test results. As middle ear effusion affects $20-30 \%$ of neonates in the neonatal intensive care unit (NICU), ${ }^{3}$ their failure rate on OAE screening is quite high. However, the prevalence of middle ear effusion in healthy newborns is only $\sim 3 \%{ }^{4}$ Thus, OAE testing is the most common screening test for newborns in well-baby nurseries because it does not require patient compliance, it is quick, and it can be performed by a technician rather than an audiologist.

The other common type of hearing assessment in neonates is the auditory brainstem evoked response (ABR). This technique involves measuring the electric field potentials generated by the brainstem in response to stimulating the cochlea with a click or short tone burst. Extraneous electrical noise not synchronised to the stimulus is removed by averaging. ABR testing is more comprehensive than OAE testing because a normal test result requires normal function of the middle ear, cochlea, auditory nerve and ascending auditory brainstem pathways (fig 1A, steps 1, 2 and 3). Automated auditory brainstem response hearing screening devices are currently used for hearing screening in many centres. These devices do not require a fully trained audiologist to perform the test and produce a dichotomous result, pass or refer (ie, fail), ${ }^{6}$ as do $\mathrm{OAE}$ tests. $\mathrm{ABR}$ and $\mathrm{OAE}$ testing modalities are complementary in that a child with abnormal auditory nerve function (auditory neuropathy) typically has normal OAEs but absent ABRs. ${ }^{7}$ Therefore, the 2007 update of the Joint Committee on Infant Hearing position statement recommended separate screening protocols for the NICU and well-baby nurseries. ${ }^{8}$ Because of the higher prevalence of auditory nerve dysfunction in infants admitted to the NICU for more than 5 days, it was recommended that these patients have $A B R$ testing as part of their hearing screening process, and not just OAE testing. ${ }^{8}$ In contrast, healthy newborns can be screened with OAE testing alone.

\section{EPIDEMIOLOGY OF NEONATAL HEARING LOSS}

Within the general population, the prevalence of permanent hearing impairment (congenital, progressive and acquired) in infants and young children in early studies from the 1980s and 1990s ranged from $0.1 \%$ to $0.2 \% .^{9-11}$ More recent studies have confirmed that this incidence has remained stable $e^{12-18}$ (table 1). However, there has been a decrease in the incidence of sensorineural hearing loss among NICU graduates when rates published in more recent reports $(0.7-1.5 \%)^{19-23}$ are compared with those from previous decades $(2.1-17.5 \%){ }^{24-28}$ This may be due to the successful preventive management of hearing loss risk factors, including quieter technology used in NICUs, better infection control, improved monitoring of oxygen supplementation and the routine measurement of serum aminoglycoside concentrations.

Recently, several large studies have focused on understanding the relationship between VLBW and hearing loss in the neonatal period and first year of life (table 2). One study compared results of newborn hearing screening tests of 1714 infants 36 weeks or older in an NICU and 25288 infants from the wellbaby nursery. ${ }^{29}$ Patients were considered to have failed their OAE screening test when either one or both ears had hearing loss. Seven percent of infants from the NICU failed the test, whereas only $1.9 \%$ of the infants from the well-baby nursery failed. Among the infants from the NICU, those with VLBW had a failure rate of $31.6 \%$. This study, however, did not address the nature of the hearing loss (conductive hearing loss associated with middle ear fluid versus sensorineural hearing loss). Also, it did not specify how many of the patients with VLBW had other coexisting risk factors for hearing loss.

Fortunately, a large percentage of VLBW patients who fail the OAE hearing screen will be found by follow-up ABR testing to have only a mild conductive hearing loss due to middle ear effusion. Although middle ear effusions are an important cause of conductive hearing loss in neonates and need to be managed, most effusions in neonates resolve spontaneously within a few weeks of birth. ${ }^{30}$ The predominant concern of this review is sensorineural hearing loss because this typically does not improve with time. It is often difficult to distinguish whether a neonate from a wellbaby nursery or NICU did not pass a hearing screening because of conductive or sensorineural hearing loss, and it is recommended that this assessment include careful diagnostic ABR testing by a qualified audiologist soon after hospital discharge. ${ }^{8}$

Another study evaluated the prevalence of hearing impairment in a cohort of 337 VLBW infants who were cared for in the NICU and survived to discharge, as well as 1205 healthy newborns. ${ }^{31}$ The OAE hearing screening test fail rate was $7.8 \%$ in the healthy control group and $12.4 \%$ for the VLBW children. On follow-up ABR testing several weeks after discharge, only $3 \%$ of the VLBW patients were found to have hearing loss. The hearing loss was conductive in $2.7 \%$ of the VLBW patients (compared with $0.06 \%$ of the healthy newborns), and one VLBW patient $(0.3 \%$ of all VLBW children) had bilateral moderate to severe sensorineural hearing loss. This study found no statistically significant differences in the prevalence of sensorineural hearing loss in the VLBW group $(0.3 \%)$, the higher weight NICU group (0.99\%) and the well-baby nursery group $(0.1 \%)$. Caveats of this study are the fact that about $30 \%$ of its VLBW population was small for gestational age but not premature, and thus this cohort was neurologically more mature than those of prior studies. This may account for better performance on the newborn hearing screening tests. Furthermore, $1.5 \%$ of the VLBW cohort was found to have abnormal prolongation of $\mathrm{ABR}$ waveform latency despite normal auditory thresholds, suggesting that even though 
Figure 1 Schematic diagram depicting the process of normal hearing in the human ear. (A) As sound pressure waves vibrate the tympanic membrane, the energy is conducted through the ossicular chain in the middle ear (step 1) to the auditory sensory organ, the cochlea. Within the cochlea, the organ of Corti contains the sensory epithelium which transduces the mechanical sound waves into electrical signals (step 2). The afferent cochlear nerve conveys the information to the brainstem, where it is processed through multiple brainstem nuclei and ultimately carried to the auditory cortex (step 3). (B) The internal structure of the cochlea. The scala tympani and scala vestibuli contain perilymph, and the scala media contains endolymph. The endocochlear potential $(+90 \mathrm{mV})$ within the scala media is maintained by the stria vascularis (SV). As the stapes footplate vibrates the perilymph fluid, the biophysical properties of the basilar membrane (BM) produce a travelling wave. The travelling wave peaks at the location of the basilar membrane tuned to the frequency of the sound stimulus. Inner hair cell (IHC) and outer hair cell $(\mathrm{OHC})$ stereociliary bundles at that location are deflected, allowing the influx of cations down a concentration gradient, which results in cell depolarisation. The afferent auditory nerve (AN) carries the signals to the brainstem.

\section{A}

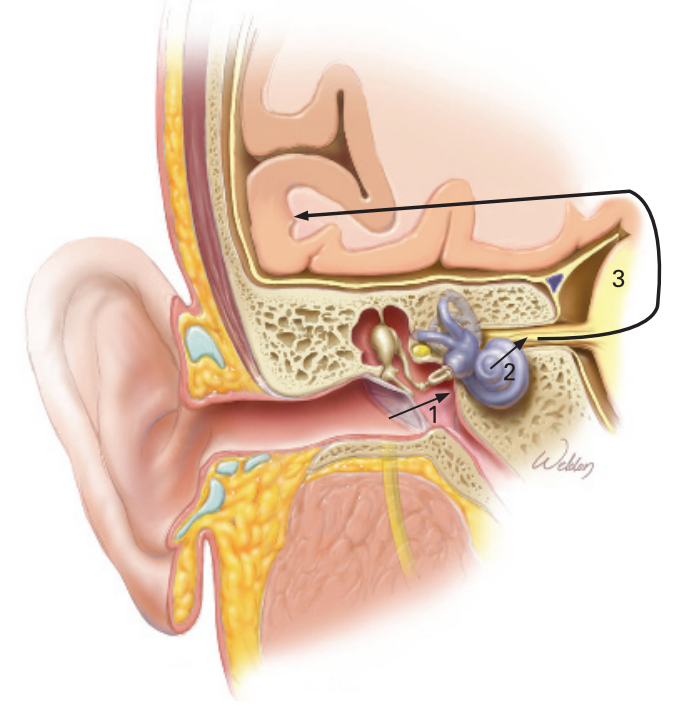

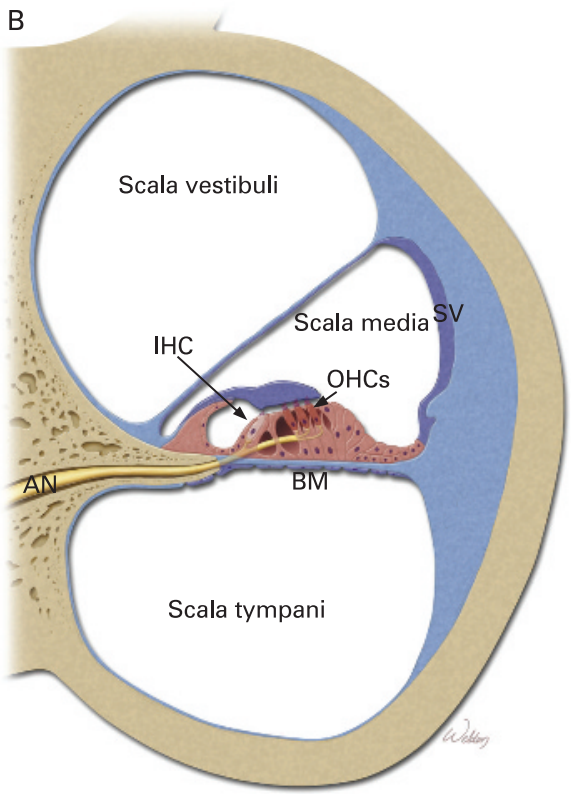

cochlear function may have been normal, there may have been abnormal ascending brainstem pathways.

Lastly, hearing testing was performed in 2995 infants at birth and at 8-12 months of age. ${ }^{9}$ In 535 infants with VLBW alone or with other risk factors, hearing testing at birth showed normal hearing in $92 \%$, transient hearing loss in $7.8 \%$, and permanent hearing loss in $2 \%$. On follow-up audiometry at 8-12 months of age, the percentage of VLBW children with permanent hearing loss was unchanged.

\section{RISK FACTORS FOR HEARING LOSS IN THE GENERAL AND VLBW NEONATAL POPULATIONS}

In 1989, Epstein and Reilly ${ }^{32}$ investigated the incidence of the known risk factors for hearing loss among all babies born in the USA. They found that $10-12 \%$ of all babies had at least one

Table 1 Incidence of congenital sensorineural hearing loss in the general population

\begin{tabular}{lll}
\hline Study & Population & Hearing loss (\%) \\
\hline Mehl \& Thomson, ${ }^{12} 2002$ & Colorado & 0.11 \\
Russ et al, ${ }^{13} 2002$ & Victoria, & $0.11-0.12$ \\
& Australia & \\
Prieve et al, ${ }^{14} 2000$ & New York & 0.19 \\
Watkin \& Baldwin, ${ }^{15} 1999$ & UK & 0.18 \\
Wessex Universal Neonatal Hearing & UK & 0.9 \\
Screening Trial Group & & \\
Maki-Torkko et al, ${ }^{17} 1998$ & Finland & 0.12 \\
Van Naarden et al, ${ }^{18} 1999$ & Georgia & 0.11 \\
\hline
\end{tabular}

established factor. The rate of sensorineural hearing loss among patients with one or more risk factors was $2-5 \%$, which is at least tenfold greater than in the general population of children. The United States Joint Committee on Infant Hearing listed VLBW as a risk factor for neonatal hearing loss in four position statements from 1973 to 1994. However, VLBW was not specifically listed as a risk factor in the statements in 2000 and 2007 (box 1). ${ }^{33}$ Instead, other risk factors commonly found in neonates with VLBW are listed. This reflects the growing understanding that VLBW by itself probably does not cause hearing loss.

A large NIH-sponsored multi-centre study conducted between 1994 and 1996 evaluated the performance of newborns on $\mathrm{OAE}$ and $\mathrm{ABR}$ hearing screening and also reported the incidence of risk factors for neonatal hearing loss. ${ }^{34}$ A total of 4478 graduates from NICUs, 353 well babies with one or more of the risk factors for hearing loss established by the Joint Committee on Infant Hearing in 1994 (which included VLBW),

Table 2 Incidence of congenital sensorineural hearing loss in infants with very low birth weight

\begin{tabular}{lll}
\hline Study & No of patients & Hearing loss (\%) \\
\hline Cone-Wesson et $a l{ }^{9} 2000$ & $11 / 535$ & 2 (Diagnostic ABR) \\
Ari-Even Roth et $a l{ }_{1}{ }^{31} 2006$ & $43 / 337$ & 12.8 (OAE screening) \\
& $6 / 337$ & 1.8 (Diagnostic ABR) \\
Korres et $a l,{ }_{,}^{29} 2005$ & $6 / 19$ & 31.6 (OAE screening)
\end{tabular}

Diagnostic $A B R$ is the gold standard for diagnosing sensorineural hearing loss. OAE screening detects both conductive and sensorineural hearing loss. $A B R$, auditory brainstem evoked response; $O A E$, otoacoustic emission. 
Box 1 Risk indicators associated with permanent

congenital, delayed-onset or progressive hearing loss in

childhood ${ }^{8}{ }^{*}$ of greater concern for delayed-onset hearing loss)

1. Caregiver concern* regarding hearing, speech, language or developmental delay.

2. Family history* of permanent childhood hearing loss.

3. Neonatal intensive care of more than 5 days or any of the following regardless of length of stay: extracorporeal membrane oxygenation, ${ }^{*}$ assisted ventilation, exposure to ototoxic drugs (gentamicin and tobramycin) or loop diuretics (furosemide), and hyperbilirubinaemia that requires exchange transfusion.

4. In utero infections, such as cytomegalovirus, ${ }^{*}$ herpes, rubella, syphilis and toxoplasmosis.

5. Craniofacial anomalies, including those that involve the pinna, ear canal, ear tags, ear pits and temporal bone anomalies.

6. Physical findings, such as a white forelock, that are associated with a syndrome known to include a sensorineural or permanent conductive hearing loss.

7. Syndromes associated with hearing loss or progressive or lateonset hearing loss, ${ }^{*}$ such as neurofibromatosis, osteopetrosis and Usher syndrome; other commonly identified syndromes include Waardenburg, Alport, Pendred, and Jervell and LangeNielson.

8. Neurodegenerative disorders, ${ }^{*}$ such as Hunter syndrome, or sensory motor neuropathies, such as Friedreich ataxia and Charcot-Marie-Tooth syndrome.

9. Culture-positive postnatal infections associated with sensorineural hearing loss, ${ }^{*}$ including confirmed bacterial and viral (especially herpes viruses and varicella) meningitis.

10. Head trauma, especially basal skull/temporal bone fracture, ${ }^{*}$ that requires hospitalisation.

11. Chemotherapy.

and 2348 well babies with no risk factors were assessed..$^{35}$ One risk factor was found in $33.2 \%$ of NICU infants, and two or more in $26.2 \%$. Within the NICU population, the most common risk factors were aminoglycoside use (44.4\%), VLBW (17.8\%), mechanical ventilation for more than 5 days $(16.4 \%)$, and low Apgar scores (13.9\%).

\section{MECHANISMS OF HEARING LOSS AND RESEARCH GAPS}

There are many different known causes of neonatal hearing loss (for a review, see Oghalai ${ }^{36}$ ). Genetic defects are thought to be responsible for about half of the $\operatorname{cases}^{37}$ and are not specifically discussed in this review. Other causes are thought to be particularly important for hearing loss in infants with VLBW. Aminoglycosides and loop diuretics have long been recognised to have the potential for ototoxicity, and strict dosing guidelines in the neonate are available in standard references. ${ }^{38}$ Other risk factors for neonatal hearing loss include noise exposure, hyperbilirubinaemia, cytomegalovirus (CMV) infection and hypoxia. ${ }^{39} 40$

\section{Aminoglycoside antibiotics}

Aminoglycosides can be the best or only choice of antibiotic for certain infections. Unfortunately, they also damage both the cochlear and vestibular organs, although they typically affect one more than the other. The two preferentially vestibulotoxic agents are gentamicin (the most widely used) and tobramycin. Aminoglycosides that are more selective to the cochlea are
Table 3 Prevalence of sensorineural hearing loss in children born with very low birth weight

\begin{tabular}{llll}
\hline Study & $\begin{array}{l}\text { No of } \\
\text { patients }\end{array}$ & $\begin{array}{l}\text { Patient age } \\
\text { (years) }\end{array}$ & $\begin{array}{l}\text { Hearing loss } \\
(\%)\end{array}$ \\
\hline Davis et $a l^{83} 2001$ & $2 / 171$ & 14 & $1.2^{*}$ \\
Doyle et $a l^{84} 1992$ & $4 / 42$ & 8 & $9.5 \dagger$ \\
\hline
\end{tabular}

${ }^{*}$ Additional central auditory processing disorder in 4/155 (2.6\%).

$\uparrow$ Additional central auditory processing disorder in 20/42 (47.6\%).

neomycin, kanamycin and amikacin. These agents produce irreversible hearing loss by causing hair cell death (fig 1A, step 2). They block ionic currents through the mechanoelectrical transduction channels in the stereocilia ${ }^{41}$ and are taken up into the hair cells through apical endocytosis. They are thought to lead to the formation of free radicals, leading to cell damage via reactive species. ${ }^{42}$ The damage to hair cells from aminoglycosides affects high-frequency hearing initially and progresses to involve lower frequencies. ${ }^{43}$ Current research efforts are focused on reducing hair cell death from aminoglycoside-induced free radicals by providing free-radical scavengers. ${ }^{44}$

In general, ototoxicity appears to correlate with duration of treatment, raised peak and trough concentrations, concurrent loop diuretics or vancomycin, underlying disease states, and previous exposure to aminoglycosides. Serum peak and trough concentrations are routinely measured, and the dose is adjusted accordingly to maintain therapeutic concentrations within the safety range. Research has shown that concentrations in the perilymph correlate with serum concentrations in rats ${ }^{45}$ and humans ${ }^{46}$ However, although high concentrations of aminoglycosides cause nearly universal vestibulocochlear damage, most patients with sensorineural hearing loss after aminoglycoside administration never had high serum concentrations of the drug. This may represent varying genetic susceptibility to aminoglycosides, and certainly genetic mutations in mitochondrial DNA have been identified in families with a history of hearing loss after administration of low doses of aminoglycosides. ${ }^{47} 48$ Therefore, monitoring of drug concentrations may give prescribing doctors a false sense of security. Monitoring and early detection of ototoxicity is best performed with serial hearing tests, particularly focused on high-frequency responses. ${ }^{49}{ }^{50}$ In 1994, a committee made up of members of the American Speech-Language-Hearing Association presented guidelines for monitoring hearing in patients treated with ototoxic drugs, ${ }^{51}$ including a first hearing test before the onset of the drug therapy, or within $72 \mathrm{~h}$ of initiation, for aminoglycosides and weekly follow-up hearing tests. Unfortunately, these tests are not often practical to perform or reliable in critically ill neonates with VLBW

Typically, a dose of $4 \mathrm{mg} / \mathrm{kg}$ gentamicin is likely to give peak drug concentrations within the desired range in neonates. Thus, some authors have stopped routinely checking serum peak drug concentrations, while still checking trough concentrations, resulting in less need for blood sampling. ${ }^{52}$ Another relatively recent advancement in the use of aminoglycosides has been once-daily dosing. This regimen is more convenient and less costly. Two recent meta-analysis articles comparing randomised controlled trials of neonates with sepsis treated with gentamicin using "once a day" and "multiple doses a day" regimens found it to be safe and efficacious, ${ }^{53}{ }^{54}$ although some authors still have concerns about ototoxicity given the higher peak concentrations reached with these regimens. ${ }^{55}{ }^{56}$ Clearly, there is a need for further research to elucidate the critical factors regarding aminoglycoside ototoxicity in order to develop better monitoring 
strategies that will permit the maximal dosing of the drug, while minimising the associated risks of toxicity.

\section{Loop diuretics}

Loop diuretics produce hearing loss by inhibiting ion transport within the stria vascularis, which reduces the electrochemical gradients that create the endocochlear potential (fig 1A, step 2). This type of hearing loss is reversible and thus is of minimal concern when treating critically ill neonates. More importantly, however, is the fact that loop diuretics potentiate the rate of aminoglycoside-induced permanent hearing loss. ${ }^{57}$ The mechanism for the aminoglycoside/loop diuretic interaction involves alterations in the blood-labyrinthine barrier, which facilitates aminoglycoside entry into the endolymphatic fluid compartment (reviewed by Ding et $a^{58}$ ). Also, loop diuretics alone may rarely cause permanent hearing loss in infants in the NICU through unknown mechanisms. ${ }^{59}$ A commonly used guideline for safe administration of furosemide or ethacrynic acid is a maximum of $2 \mathrm{mg} / \mathrm{kg} /$ dose every $12 \mathrm{~h}$. However, a recent study showed that half this dose is associated with sensorineural hearing loss in neonates with hypoxaemia. ${ }^{60}$ Thus, major research gaps exist in our understanding of the pathophysiology of hearing loss secondary to loop diuretics.

\section{Noise-induced hearing loss}

Exposure to the constant background noise generated by contemporary life-support equipment in the NICU can produce hearing loss. ${ }^{61}$ The initial sign is outer hair cell damage (fig $1 \mathrm{~A}$, step 2). This may occur because the stereocilia of neonates are attached to the tectorial membrane and thus may be more easily over-stimulated. In theory, if all of the rest of the cochlea continues to function normally, this should only produce a partial hearing loss $(\sim 60 \mathrm{~dB}){ }^{2}$ However, many studies point out that noise trauma can also produce damage to the inner hair cells, stria vascularis, spiral ganglion cells and supporting cells. Recent studies suggest that this could be due to free-radical formation. ${ }^{62}$ As hair cells from the human cochlea lack the ability to regenerate, severe acoustic trauma or prolonged noise exposure may lead to complete sensorineural hearing loss. Similar to aminoglycoside ototoxicity, current research efforts are focusing on reducing free-radical damage using scavenging agents. $^{63}$ Even more importantly, preventive bioengineering efforts continue to focus on reducing the intensity of the noise produced by machines in the NICU.

\section{Hyperbilirubinaemia}

Hyperbilirubinaemia can cause selective damage to the brainstem auditory nuclei and may also damage the auditory nerve and spiral ganglion cells ${ }^{64}$ (fig $1 \mathrm{~A}$, step 3 ). It does this by interfering with neuronal intracellular calcium homoeostasis. ${ }^{64}$ In contrast, the organ of Corti and thalamocortical auditory pathways appear to be unaffected by bilirubin. Clinically, a common form of hearing loss caused by hyperbilirubinaemia is auditory neuropathy. Thus, OAE screening is normal but ABR testing is abnormal. This is one type of sensorineural hearing loss that may occasionally spontaneously resolve, typically by 12 months of age in our experience.

In preterm infants, the relationship between hyperbilirubinaemia and hearing loss is significant and is modulated by other risk factors. De Vries et al ${ }^{65}$ found that, among preterm infants with high bilirubin concentrations ( $14 \mathrm{mg} / \mathrm{dL}$ or greater), those with birth weight of $\leqslant 1500 \mathrm{~g}$ carry a higher risk of deafness than their healthy counterparts with birth weight $>1500 \mathrm{~g}$.
Furthermore, among the high-risk patients, the mean duration of hyperbilirubinaemia was significantly longer in the deaf infants, and they appeared to have a greater number of acidotic episodes while they were hyperbilirubinaemic.

In full-term infants with birth weight $>2500 \mathrm{~g}$, the concentration of bilirubin required for ototoxicity to occur remains less clear. One study compared hearing in 99 full-term neonates (>37 weeks' gestation, birth weight $>2500 \mathrm{~g}$ ) with moderate hyperbilirubinaemia (mean maximum concentration of $18.9 \mathrm{mg} / \mathrm{dl}$ ), severe hyperbilirubinaemia (mean maximum concentration of $21.7 \mathrm{mg} / \mathrm{dl}$ ), and super hyperbilirubinaemia (mean maximum concentration of $26.9 \mathrm{mg} / \mathrm{dl}$ ) in the absence of congenital or metabolic anomalies, asphyxia, sepsis, meningitis, or other brain anomalies. ${ }^{66}$ This study found no differences in the prevalence of hearing loss at initial or follow-up hearing assessments between the groups with different concentrations.

\section{CMV}

Although CMV is known to cause white matter changes in the central nervous system, it causes sensorineural hearing loss by affecting the cochlea (fig 1A, step 2). Unfortunately, its pathophysiological mechanisms are poorly understood. The guinea pig is the only known animal model in which CMV can cross the placental barrier and infect the fetus. ${ }^{67}$ Injection of CMV into 5-week-old pregnant guinea pigs was found to result in severe fetal labyrinthitis. ${ }^{68}$ Immunohistochemistry detected viral infection of the endothelial cells surrounding the perilymph and of the spiral ganglion cells, but not within the organ of Corti. Loss of the spiral ganglion cells could partially account for the hearing loss in these patients. Clinically, however, the lack of OAEs suggests a cochlear mechanism for the hearing loss in humans. Therefore, the guinea pig model is not necessarily representative of the human condition and this constitutes a major research gap in this area. In terms of using human tissue to study the disease pathophysiology, the most obvious technique is through the study of archival temporal bones. However, there is a paucity of temporal bones from young children deafened because of congenital CMV infection. One case report describes loss of cochlear hair cells and strial atrophy along the entire length of the basilar membrane of the cochlea in an autopsy specimen of a 14-year-old girl with a history of congenital CMV infection. ${ }^{69}$

\section{Hypoxia}

Hypoxia has a strong association with hearing loss. Adequate oxygenation and perfusion are essential for normal cochlear function. ${ }^{70}$ In newborn infants with hypoxia or asphyxia, the spiral ganglion cells appear to be affected first ${ }^{72}$ (fig 1A, step 3). More severe hypoxia may cause irreversible cellular damage to the cochlea, particularly to the outer hair cells and stria vascularis (fig 1A, step 2). However, there is no clear threshold level of hypoxia defining the point at which hearing is at risk. Also, there is a definite variability in the susceptibility of patients to develop hearing loss after hypoxia. The reasons for this are unclear. In addition to the effects of hypoxia, infants with respiratory failure are often treated with hyperventilation and/or alkalinisation, which may further decrease the oxygenation and perfusion of the cochlea and auditory pathway, leading to hearing loss. ${ }^{73}$ Although the prevalence of sensorineural hearing loss in NICU graduates is $1-3 \%,{ }^{74}$ one study found that $>50 \%$ of survivors of severe neonatal respiratory failure had sensorineural hearing loss at 4 years of age. ${ }^{75}$ Many of these patients did not begin to develop hearing loss until 2-4 years of 
age. ${ }^{76}$ The pathophysiology behind the delayed nature of the hearing loss is unclear.

In addition, the use of extracorporeal membrane oxygenation has been found to increase the prevalence of sensorineural hearing loss among NICU survivors. In these patients, the prevalence of sensorineural hearing loss is in the range 3$26 \% .^{77}$ Fligor et $a 7^{78}$ found that, among children who had received extracorporeal membrane oxygenation, $\sim 70 \%$ with hearing loss had progressive worsening. Also, 35\% of the children developed hearing loss in a delayed fashion, supporting the need for close monitoring of hearing throughout childhood in these patients.

\section{LONG-TERM EFFECTS OF HEARING LOSS IN CHILDREN WITH VLBW}

The long-term consequences of neonatal and infant hearing loss are now well recognised. It has become evident that hearing impairment early in life affects communication, cognition, behaviour, social and emotional development, academic outcomes and later vocational opportunities. ${ }^{79-81}$ Also, recent studies show that hearing loss can develop in a delayed fashion, and failure to diagnose this condition places children at an academic disadvantage. Fortnum et al ${ }^{82}$ reported that the prevalence of permanent childhood hearing impairment continues to rise until the age of 9 years and may be as high as 205 per 100000 for the general population. The relationship between VLBW and progressive or delayed-onset sensorineural hearing loss remains poorly understood. To date, few data correlating birth weight with progressive hearing loss are available (table 3).

One study found no significant differences in the rates of hearing impairment between children with VLBW and those with normal birth weight at 14 years of age. ${ }^{83}$ However, this study also measured central auditory processing and reported that children with VLBW had decreased ability to recall auditory information when their memory was overloaded with long sentences $(2.6 \%)$. This was associated with poorer IQ, reading and spelling scores, as well as behavioural difficulties such as antisocial behaviour and feelings of social rejection. Another study has reported that the prevalence of central auditory processing disorder was even higher in VLBW children when they were only 8 years old $(47.6 \%) .{ }^{84}$ Thus, the real impact of VLBW on the peripheral and central aspects of hearing by the time of early adulthood is not known at this time. The 2007 Joint Committee on Infant Hearing position statement clearly states that "Infants who pass the neonatal screening but have a risk factor should have at least one diagnostic audiology assessment by 24 to 30 months of age". 8 However, these studies suggest that central auditory processing disorders may be present. Unfortunately, these problems would be missed by routine audiometric examination, which typically does not assess central auditory processing.

\section{CONCLUSIONS}

The prevalence of failed hearing screening in neonates with VLBW is significantly higher than in neonates with normal birth weight because they experience higher rates of transient middle ear fluid accumulation and conductive hearing loss. This temporary hearing loss usually resolves within weeks of discharge from the hospital. The extent to which VLBW alone increases the prevalence of sensorineural hearing impairment in the early neonatal period remains unclear. However, these patients are commonly exposed to other risk factors for hearing loss such as ototoxic drugs, hypoxia and hyperbilirubinaemia, which may lead to early or delayed-onset sensorineural hearing loss as well as progression of a mild pre-existing sensorineural hearing loss years after hospital discharge. Furthermore, the presence of hearing loss in the early years of life can have additional negative effects on central auditory processing and intellectual functioning. Thus, long-term careful monitoring for hearing loss and the appropriate audiological management of hearing loss in children with VLBW as well as those with risk factors for hearing loss is essential.

Acknowledgements: We appreciate the critical feedback provided by Drs Ann Stark, Robert Lasky and Robert Fanning on earlier versions of the article Artwork by Scott Weldon.

Funding: $\mathrm{NIH}$.

Competing interests: None.

\section{REFERENCES}

1. Ehrenhaft PM, Wagner JL, Herdman RC. Changing prognosis for very low birth weight infants. Obstet Gynecol 1989;74:528-35.

2. Oghalai JS. The cochlear amplifier: augmentation of the traveling wave within the inner ear. Curr Opin Otolaryngol Head Neck Surg 2004;12:431-8.

3. Balkany TJ, Downs MP, Jafek BW, et al. Otologic manifestations of Down's syndrome. Surg Forum 1978;29:582-5.

4. Doyle KJ, Kong YY, Strobel K, et al. Neonatal middle ear effusion predicts chronic otitis media with effusion. Otol Neurotol 2004;25:318-22.

5. van Straaten HL, Tibosch $\mathrm{CH}$, Dorrepaal C, et al. Efficacy of automated auditory brainstem response hearing screening in very preterm newborns. J Pediatr 2001;138:674-8.

6. van Straaten HL, Hille ET, Kok JH, et al. Implementation of a nation-wide automated auditory brainstem response hearing screening programme in neonatal intensive care units. Acta Paediatr 2003;92:332-8.

7. Berg AL, Spitzer JB, Towers HM, et al. Newborn hearing screening in the NICU: profile of failed auditory brainstem response/passed otoacoustic emission. [Erratum in Pediatrics 2006;117:997.] Pediatrics 2005;116:933-8.

8. Hearing JCol. Year 2007 position statement: principles and guidelines for early hearing detection and intervention programs. Pediatrics 2007;120:989-21.

9. Cone-Wesson B, Vohr BR, Sininger YS, et al. Identification of neonatal hearing impairment: infants with hearing loss. Ear Hear 2000;21:488-507.

10. Parving A. Detection of the infant with congenital/early acquired hearing disability. Acta Otolaryngol Supp/ 1991;482:111-16 [discussion 7].

11. Davis A, Wood S. The epidemiology of childhood hearing impairment: factor relevant to planning of services. Br J Audiol 1992;26:77-90.

12. Mehl AL, Thomson V. The Colorado newborn hearing screening project, 1992-1999: on the threshold of effective population-based universal newborn hearing screening. [See comment.] Pediatrics 2002;109:E7.

13. Russ SA, Rickards F, Poulakis Z, et al. Six year effectiveness of a population based two tier infant hearing screening programme. Arch Dis Child 2002;86:245-50.

14. Prieve B, Dalzell L, Berg A, et al. The New York State universal newborn hearing screening demonstration project: outpatient outcome measures. [See comment.] Ear Hear 2000;21:104-17.

15. Watkin PM, Baldwin M. Confirmation of deafness in infancy. Arch Dis Child 1999:81:380-9.

16. Wessex Universal Neonatal Hearing Screening Trial Group. Controlled trial of universal neonatal screening for early identification of permanent childhood hearing impairment. Lancet 1998;352:1957-64.

17. Maki-Torkko EM, Lindholm PK, Vayrynen MR, et al. Epidemiology of moderate to profound childhood hearing impairments in northern Finland. Any changes in ten years? Scand Audiol 1998;27:95-103

18. Van Naarden K, Decoufle P, Caldwell K. Prevalence and characteristics of children with serious hearing impairment in metropolitan Atlanta, 1991-1993. [See comment.] Pediatrics 1999;103:570-5.

19. Smyth V, Scott J, Tudehope D. Auditory brainstem evoked response inter-peak latencies in very low birthweight infants. Int J Pediatr Otorhinolaryngol 1988;16:69-76.

20. Veen S, Sassen ML, Schreuder AM, et al. Hearing loss in very preterm and very low birthweight infants at the age of 5 years in a nationwide cohort. Int $J$ Pediatr Otorhinolaryngol 1993;26:11-28.

21. Meyer C, Witte J, Hildmann A, et al. Neonatal screening for hearing disorders in infants at risk: incidence, risk factors, and follow-up. Pediatrics 1999;104:900-4.

22. Perrott S, Dodds L, Vincer M. A population-based study of prognostic factors related to major disability in very preterm survivors. J Perinatol 2003;23:111-16.

23. Oghalai JS, Chen L, Brennan ML, et al. Neonatal hearing loss in the indigent. The Laryngoscope 2002;112:281-6.

24. Abramovich SJ, Gregory S, Slemick M, et al. Hearing loss in very low birthweight infants treated with neonatal intensive care. Arch Dis Child 1979;54:421-6. 
25. Bergman I, Hirsch RP, Fria TJ, et al. Cause of hearing loss in the high-risk premature infant. J Pediatr 1985;106:95-101.

26. Bradford BC, Baudin J, Conway MJ, et al. Identification of sensory neural hearing loss in very preterm infants by brainstem auditory evoked potentials. Arch Dis Child 1985;60:105-9.

27. Salamy A, Eldredge L, Tooley WH. Neonatal status and hearing loss in high-risk infants J Pediatr 1989:114:847-52.

28. Jiang ZD, Brosi DM, Wilkinson AR. Hearing impairment in preterm very low birthweight babies detected at term by brainstem auditory evoked responses. Acta Paediatr 2001:90:1411-15.

29. Korres S, Nikolopoulos TP, Komkotou V, et al. Newborn hearing screening: effectiveness, importance of high-risk factors, and characteristics of infants in the neonatal intensive care unit and well-baby nursery. Otol Neurotol 2005:26:1186-90.

30. Rosenfeld RM, Bluestone CD. Evidence-based otitis media. 2nd edn. Hamilton, Ont, Lewiston, NY: BC Decker, 2003

31. Ari-Even Roth D, Hildesheimer M, Maayan-Metzger A, et al. Low prevalence of hearing impairment among very low birthweight infants as detected by universal neonatal hearing screening. Arch Dis Child Fetal Neonatal Ed 2006:91:F257-62.

32. Epstein S, Reilly JS. Sensorineural hearing loss. Pediatr Clin North Am 1989;36:1501-20.

33. Joint Committee on Infant Hearing, American Academy of Audiology, American Academy of Pediatrics, American Speech-Language-Hearing Association, and Directors of Speech and Hearing Programs in State Health and Welfare Agencies. Year 2000 position statement: principles and guidelines for early hearing detection and intervention programs. Pediatrics 2000;106:798-817.

34. Norton SJ, Gorga MP. Widen JE, et al. Identification of neonatal hearing impairment: summary and recommendations. Ear Hear 2000;21:529-35.

35. Vohr BR, Widen JE, Cone-Wesson B, et al. Identification of neonatal hearing impairment: characteristics of infants in the neonatal intensive care unit and wellbaby nursery. Ear Hear 2000;21:373-82.

36. Oghalai JS. Cochlear hearing loss. In: Jackler RK, Brackmann DE, eds. Neurotology. 2nd edn. Philadelphia: Mosby, 2005:589-606.

37. Steel KP, Kros CJ. A genetic approach to understanding auditory function. Nat Genet 2001:27:143-9.

38. Young TE, Magnum OB. Neofax: a manual of drugs used in neonatal care. 20th edn. Thomson Healthcare, 2007.

39. Marlow ES, Hunt LP, Marlow N. Sensorineural hearing loss and prematurity. Arch Dis Child Fetal Neonatal Ed 2000;82:F141-4.

40. Fanaroff JM, Wilson-Costello DE, Newman NS, et al. Treated hypotension is associated with neonatal morbidity and hearing loss in extremely low birth weight infants. Pediatrics 2006;117:1131-5.

41. Forge A, Schacht J. Aminoglycoside antibiotics. Audiol Neurootol 2000;5:3-22.

42. Rotstein C, Mandell L. Clinical aminoglycoside ototoxicity. In: Roland PS, Rutka JA, eds. Ototoxicity. Hamilton, Ont: BC Decker, 2004:82-92.

43. Fausti SA, Henry JA, Helt WJ, et al. An individualized, sensitive frequency range for early detection of ototoxicity. Ear Hear 1999;20:497-505.

44. Kawamoto K, Sha SH, Minoda R, et al. Antioxidant gene therapy can protect hearing and hair cells from ototoxicity. Mol Ther 2004:9:173-81.

45. Becvarovski Z, Michaelides EM, Kartush JM, et al. Rapid elevation of gentamicin levels in the human labyrinth following intravenous administration. Laryngoscope 2002:112:1163-5.

46. Rizzi MD, Hirose K. Aminoglycoside ototoxicity. Curr Opin Otolaryngol Head Neck Surg 2007:15:352-7.

47. Casano RA, Johnson DF, Bykhovskaya $Y$, et al. Inherited susceptibility to aminoglycoside ototoxicity: genetic heterogeneity and clinical implications Am J Otolaryngol 1999;20:151-6.

48. Guan MX. Molecular pathogenetic mechanism of maternally inherited deafness. Ann N Y Acad Sci 2004;1011:259-71.

49. Stavroulaki P, Apostolopoulos N, Dinopoulou D, et al. Otoacoustic emissions: an approach for monitoring aminoglycoside induced ototoxicity in children. Int J Pediatr Otorhinolaryngol 1999;50:177-84.

50. Fausti SA, Larson VD, Noffsinger D, et al. High-frequency audiometric monitoring strategies for early detection of ototoxicity. Ear Hear 1994;15:232-9.

51. American Speech-Language-Hearing Association. Audiologic managment of individuals receiving cochleotoxic drug therapy. Guidelines for the audiologic management of individuals receiving cochleotoxic drug therapy. ASHA Supplement 1994:36(Suppl 12):11-19.

52. Bajaj M, Palmer K. Gentamicin usage in newborns: an audit [comment]. Arch Dis Child 2003;88:645.

53. Rao SC, Ahmed M, Hagan R. One dose per day compared to multiple doses per day of gentamicin for treatment of suspected or proven sepsis in neonates. Cochrane Database Syst Rev 2006;(1):CD005091.

54. Nestaas E, Bangstad HJ, Sandvik L, et al. Aminoglycoside extended interval dosing in neonates is safe and effective: a meta-analysis. [See comment.] Arch Dis Child Fetal Neonatal Ed 2005:90:F294-300.
55. Best EJ, Palasanthiran P, Gazarian M. Extended-interval aminoglycosides in children: more guidance is needed [comment]. Pediatrics 2005;115:827-8; author reply 8 .

56. Loughnan PM. Single daily dose aminoglycosides in the neonatal period appear to be effective: but are they safe? [comment]. Arch Dis Child Fetal Neonatal Ed 2006;91:F156.

57. Humes HD. Insights into ototoxicity. Analogies to nephrotoxicity. Ann N Y Acad Sci 1999:884:15-18.

58. Ding D, McFadden SL, Browne RW, et al. Late dosing with ethacrynic acid can reduce gentamicin concentration in perilymph and protect cochlear hair cells. Hearing Res 2003;185:90-6.

59. Brown DR, Watchko JF, Sabo D. Neonatal sensorineural hearing loss associated with furosemide: a case-control study. Dev Med Child Neurol 1991;33:816-23.

60. Robertson CM, Tyebkhan JM, Peliowski A, et al. Ototoxic drugs and sensorineural hearing loss following severe neonatal respiratory failure. Acta Paediatr 2006;95:214-23.

61. Williams AL, van Drongelen W, Lasky RE. Noise in contemporary neonatal intensive care. J Acoust Soc Am 2007:121:2681-90.

62. Le Prell CG, Yamashita D, Minami SB, et al. Mechanisms of noise-induced hearing loss indicate multiple methods of prevention. Hear Res 2007;226:22-43.

63. Kramer S, Dreisbach L, Lockwood J, et al. Efficacy of the antioxidant Nacetylcysteine (NAC) in protecting ears exposed to loud music. J Am Acad Audiol 2006:17:265-78.

64. Shapiro SM, Nakamura H. Bilirubin and the auditory system. J Perinatol 2001:21(Suppl 1):S52-5; discussion S9-62.

65. De Vries LS, Lary S, Whitelaw AG, et al. Relationship of serum bilirubin levels and hearing impairment in newborn infants. Early Hum Dev 1987;15:269-77.

66. Wong V, Chen WX, Wong KY. Short- and long-term outcome of severe neonatal nonhemolytic hyperbilirubinemia. J Child Neurol 2006;21:309-15.

67. Schleiss MR. Nonprimate models of congenital cytomegalovirus (CMV) infection: gaining insight into pathogenesis and prevention of disease in newborns. ILAR J 2006; $47: 65-72$

68. Katano H, Sato Y, Tsutsui $Y$, et al. Pathogenesis of cytomegalovirus-associated labyrinthitis in a guinea pig model. Microbes Infect 2007:9:183-91.

69. Rarey KE, Davis LE. Temporal bone histopathology 14 years after cytomegalic inclusion disease: a case study. Laryngoscope 1993:103:904-9.

70. Haupt $\mathbf{H}$, Scheibe F, Ludwig C. Changes in cochlear oxygenation, microcirculation and auditory function during prolonged general hypoxia. Eur Arch Otorhinolaryngol 1993:250:396-400.

71. Sohmer H, Freeman S, Malachi S. Multi-modality evoked potentials in hypoxaemia. Electroencephalogr Clin Neurophysiol 1986;64:328-33.

72. Koyama S, Kaga K, Sakata H, et al. Pathological findings in the temporal bone of newborn infants with neonatal asphyxia. Acta Otolaryngol 2005;125:1028-32.

73. Rais-Bahrami K, Majd M, Veszelovszky E, et al. Use of furosemide and hearing loss in neonatal intensive care survivors. Am J Perinatol 2004;21:329-32.

74. National Institutes of Health Consensus Development Conference

Statement. Early identification of hearing impairment in infants and young children. Int J Pediatr Otorhinolaryngol 1993;27:215-27.

75. Robertson CM, Tyebkhan JM, Hagler ME, et al. Late-onset, progressive sensorineural hearing loss after severe neonatal respiratory failure. Otol Neurotol 2002:23:353-6

76. Robertson CM, Tyebkhan JM, Peliowski A, et al. Ototoxic drugs and sensorineural hearing loss following severe neonatal respiratory failure. Acta Paediatr 2006;95:214-23

77. Lasky RE, Wiorek L, Becker TR. Hearing loss in survivors of neonatal extracorporeal membrane oxygenation (ECMO) therapy and high-frequency oscillatory (HFO) therapy. J Am Acad Audiol 1998:9:47-58.

78. Fligor BJ, Neault MW, Mullen $\mathrm{CH}$, et al. Factors associated with sensorineural hearing loss among survivors of extracorporeal membrane oxygenation therapy. Pediatrics 2005:115:1519-28.

79. Karchmer MA, Allen TE. The functional assessment of deaf and hard of hearing students. Am Ann Deaf 1999:144:68-77.

80. Pierson SK, Caudle SE, Krull KR, et al. Cognition in children with sensorineural hearing loss: etiologic considerations. Laryngoscope 2007;117:1661-5.

81. Kushalnagar P, Krull K, Hannay J, et al. Intelligence, parental depression, and behavior adaptability in deaf children being considered for cochlear implantation. J Deaf Stud Deaf Educ 2007:12:335-49.

82. Fortnum HM, Summerfield AO, Marshall DH, et al. Prevalence of permanent childhood hearing impairment in the United Kingdom and implications for universal neonatal hearing screening: questionnaire based ascertainment study. BMJ 2001;323:536-40.

83. Davis NM, Doyle LW, Ford GW, et al. Auditory function at 14 years of age of verylow-birthweight. Dev Med Child Neurol 2001:43:191-6.

84. Doyle LW, Keir E, Kitchen WH, et al. Audiologic assessment of extremely low birth weight infants: a preliminary report. Pediatrics 1992:90:744-9. 